\title{
AVANCES DEL MEJORAMIENTO GENÉTICO PARTICIPATIVO DEL FRIJOL EN CUBA 1
}

\author{
Rodobaldo Ortiz-Pérez ${ }^{2}$, Humberto Ríos-Labrada ${ }^{2}$, Sandra Miranda-Lorigados ${ }^{2}$, Manuel Ponce-Brito ${ }^{2}$, \\ Edilio Quintero-Fernández ${ }^{3}$, Orlando Chaveco-Pérez ${ }^{4}$
}

\section{RESUMEN}

Avances del mejoramiento genético participativo del frijol en Cuba. Las actividades de Fitomejoramiento Participativo fueron conducidas con agricultores del municipio de San Antonio de los Baños de la provincia La Habana y campesinos de la comunidad la Palma de la provincia de Pinar del Río durante 2001 al 2004. En la fase de diagnóstico en las áreas de intervención del proyecto, más del $80 \%$ de los agricultores no utilizaban la semilla del sistema formal; por lo tanto, las variedades obtenidas por los programas de mejoramiento no llegaban a la mayoría de los agricultores. Se buscó un mecanismo alternativo para introducir diversidad genética a las localidades mediante las ferias de diversidad, cuyo objetivo principal ha estado dirigido a facilitar el flujo de semilla de los institutos de investigaciones hacia el agricultor y viceversa. Posterior al desarrollo de las ferias de diversidad se continúa con la experimentación campesina en fincas y cooperativas, desarrollándose una amplia red experimental difícil de lograrse sin la participación de los productores. La experimentación campesina, el aumento de la eficiencia en la finca, incluyendo el aumento del rendimiento de sus parcelas, el aumento de la diversidad por el uso de mayor número de variedades, y una mayor proporción de área dedicada a estas variedades; todo lo cual redunda en un mejoramiento de la vida del campesino y su familia.

Palabras clave: Fitomejoramiento participativo, semilla local, ferias de biodiversidad, experimentación campesina, frijol (Phaseolus vulgaris L.).

\begin{abstract}
Progress in participatory plant breeding of common beans in Cuba. Participatory Plant Breeding activities were performed with the support of farmers from both San Antonio de los Baños Municipality in Habana province and La Palma community in Pinar del Río province between 2001 and 2004. In the diagnosis phase, it was observed that more than $80 \%$ of farmers did not use the seeds provided by the official system in the target areas of the project. Therefore, the varieties obtained by conventional breeding programs did not reach most farmers. An alternative mechanism was developed to introduce genetic diversity to several localities, with the objective of facilitating seed transfer from research institutions to the farmer and viceversa. After conducting diversity fairs, rural experimentation will follow in farms and cooperatives, developing a broad experimental network difficult to achieve by any breeding program that does not consider producer's participation. Rural experimentation, increases in diversity due to the use of greater number of varieties, and larger proportion of areas dedicated to the cultivation of these species resulted in the improvement of the wellbeing of the farmers and their families.
\end{abstract}

Palabras clave: Participatory plant breeding, local seed, biodiversity fairs, farmer experimentation, common bean (Phaseolus vulgaris L.).

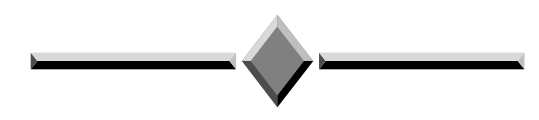

1 Recibido: 29 de setiembre, 2005. Aceptado: 13 de julio, 2006.

2 Instituto Nacional de Ciencias Agrícolas (INCA). Correos electrónicos: rortiz@inca.edu.cu; burumbun@yahoo.com; sandra@inca.edu.cu; ponce@inca.edu.cu

3 Centro de Investigaciones Agropecuarias, Universidad Central (CIAT). Correo electrónico: equintero@usc.edu.cu

4 Estación Territorial de Investigaciones Agropecuarias de Holguín (ETIAH). Correo electrónico: granos@holguin.inf.cu 


\section{INTRODUCCIÓN}

Con la entrada al periodo especial en Cuba, por la caída del campo socialista europeo a finales de la década de los años 80, se observó un deterioro de toda la producción de semilla del sistema formal; este sistema se encontraba en la imposibilidad de producir semilla para satisfacer en cantidad y calidad las demandas de los agricultores. Paralelamente, se observaron limitaciones en el fitomejoramiento convencional para satisfacer en materia de diversidad varietal, las exigencias de las diversas condiciones agroecológicas generadas por la falta de insumos para la producción de alimentos: adicionalmente, se llegó a un deterioro del sistema convencional de fitomejoramiento, por falta de recursos para su ejecución.

El estado cubano trabajó con intensidad en el desarrollo de una agricultura de bajos insumos con la utilización tracción animal, bajo índice de gasto de combustible, no utilización de fertilizantes y plaguicidas químicos, amplio uso de abonos alternativos orgánicos, controles y reguladores de plagas y enfermedades, dividió las empresas estatales en cooperativas de producción agropecuarias, entregó parcelas y fincas a nuevos agricultores, y desarrolló al máximo la agricultura urbana.

Como parte de esta respuesta a las nuevas condiciones, durante 1999 se diseñó un proyecto de fitomejoramiento participativo (FP) por integrantes del Departamento de Genética y Mejoramiento del Instituto Nacional de Ciencias Agrícolas (INCA), con el objetivo de aumentar el rendimiento y calidad de las cosechas del maíz y frijol común, sobre la base del incremento de la diversidad varietal y el fomento de los grupos de investigación campesina (GICs), como célula de selección, intercambio, conservación y multiplicación de semillas de variedades mejoradas a nivel local.

En el presente trabajo se presentan los avances logrados en la aplicación del fitomejoramiento participativo del frijol en Cuba.

\section{MATERIALES Y MÉTODOS}

Las actividades de FP que se presentan, fueron conducidas con agricultores del municipio de San Antonio de los Baños de la provincia La Habana y campesinos de la comunidad la Palma de la provincia de Pinar del Río. Los agricultores participantes en la Habana están organizados en dos cooperativas de producción agropecuarias (CPA) con agrupamiento de sus tierras con una junta elegida por los cooperativistas y con una extensión de 180 ha y 67 socios en la CPA Jorge Dimitrov y una extensión de 470 ha y 109 socios en la CPA Gilberto León. Los de Pinar del Río están organizados en una cooperativa de créditos y servicios (CSS) de la Palma, donde cada agricultor es dueño y administra su finca que tiene una extensión de cuatro a 60 ha, en esta localidad se utilizó el trabajo de ocho campesinos. Además se presentan datos de la intervención del proyecto a nivel nacional, que complementan la información de los casos de estudio.

Las herramientas utilizadas en el proceso investigativo fueron:

Diagnóstico sobre aspectos relacionados con los sistemas de semilla en los lugares de intervención del proyecto

En las CPA de la Habana se diseñó una encuesta sobre los cultivos de frijol y datos generales de cada CPA. En el municipio la Palma, Pinar del Río se identificaron las fincas donde se manejaba una diversidad representativa de la diversidad de frijol de la comunidad El Tejar La Jocuma. Para esto se realizaron encuestas y visitas a las parcelas de 23 campesinos dueños de fincas, lo que representó el $47 \%$ de las fincas de la comunidad.

Se efectuaron colectas en diversas localidades para determinar la diversidad genética utilizada por los campesinos, bajo los sistemas de semilla locales

Con el objetivo de evaluar el impacto de las colectas en cuanto a su magnitud a nivel de país, se procesó la información existente del 2004, con todas las accesiones colectadas y el uso del germoplasma "ex situ", donado por otras instituciones para este programa de FP.

Establecimiento de Ferias de Diversidad como herramienta para introducir diversidad genética a las localidades donde los agricultores participantes podrían seleccionar un grupo de variedades

Una caracterización de las Ferias de Diversidad aparece en una metodología conformada y publicada 
para su divulgación y uso por centros de investigación cubanos y de otros países (De la Fe et al. 2003).

En siembras tardías se diseñó una feria de frijol, en la cual, se sembraron 80 accesiones de frijoles, incluyendo 19 variedades comerciales y 20 variedades pre-comerciales cubanas, 20 variedades del banco de germoplasma cubano donadas por el Instituto de Investigaciones Fundamentales de la Agricultura Tropical (INIFAT), y 21 accesiones colectadas en La Palma. El cultivo se mantuvo con tres riegos de supervivencia, sin control fitosanitario y sin fertilización. Los materiales fueron distribuidos en dos bloques, donde cada línea estaba representada por cuatro surcos de cuatro metros de largo (cada variedad estuvo identificada por un orden numérico consecutivo). Los lotes al momento de la feria estaban en total madurez fisiológica.

La feria se celebró el viernes 6 de abril del 2001 en áreas agrícolas del INCA. Se seleccionaron, entre los participantes, ocho campesinos de las CPA de La Habana y ocho de cooperativas de crédito y servicio del municipio de La Palma, Pinar del Río. Cada campesino pudo seleccionar las siete líneas que más le gustaron. Al finalizar el proceso selectivo por los campesinos participantes en la feria, se les informó los nombres de las variedades que ellos habían escogido.

Con el objetivo de evaluar el impacto de las ferias en cuanto a la difusión de materiales y a número de participantes, se procesó la información existente de las ferias de frijol ejecutadas en el país hasta finales del 2004.

La experimentación campesina con base en los materiales seleccionados en las ferias de diversidad: selección de materiales promisorios en sus fincas y su uso potencial por los campesinos.

Las variedades seleccionadas por los campesinos en las Ferias de Frijol fueron entregadas a las CPA y CCS en agosto del 2001.

Primera etapa selectiva de los frijoles en las CPAs (Cuadro 1): En siembras tempranas, se sembraron las 13 variedades nuevas y la variedad negra local en la CPA G. León, en surcos de diferentes dimensiones según la cantidad de semilla entregada: cada tres surcos de frijol fueron intercalados a $90 \mathrm{~cm}$ entre surcos de plátano "Burro nuevo". El 27 de diciembre
Cuadro 1. Variedades de frijol evaluadas en las CPA de la Habana en época temprana y tardía. San Antonio de los Baños, Cuba. 2001 y 2002.

\begin{tabular}{|c|c|c|c|c|}
\hline \multirow{3}{*}{\begin{tabular}{c}
\multicolumn{1}{c}{ CPA } \\
\multicolumn{1}{c}{ Época } \\
F. Siembra \\
Variedades
\end{tabular}} & \multicolumn{2}{|c|}{ Gilberto León } & \multicolumn{2}{|c|}{ Jorge Dimitrov } \\
\hline & $\begin{array}{c}\text { Temprana } \\
26 / 9 / 01\end{array}$ & $\begin{array}{c}\text { Tardía } \\
\text { 18/01/02 }\end{array}$ & $\begin{array}{c}\text { Temprana } \\
2 / 10 / 01\end{array}$ & $\begin{array}{c}\text { Tardía } \\
\text { 14/01/02 }\end{array}$ \\
\hline & \multicolumn{4}{|c|}{ Utilizadas } \\
\hline 1 & $X$ & $\mathrm{X}$ & $X$ & $\mathrm{X}$ \\
\hline 2 & & $X$ & $X$ & $X$ \\
\hline 4 & $\mathrm{X}$ & $\mathrm{X}$ & & $\mathrm{X}$ \\
\hline 9 & & $X$ & $X$ & $X$ \\
\hline 41 & $\mathrm{X}$ & $\mathrm{X}$ & & $\mathrm{X}$ \\
\hline 42 & $X$ & $\mathrm{X}$ & $X$ & $X$ \\
\hline 44 & $\mathrm{X}$ & $\mathrm{X}$ & $\mathrm{X}$ & $\mathrm{X}$ \\
\hline 47 & $\mathrm{X}$ & $\mathrm{X}$ & & $\mathrm{X}$ \\
\hline 48 & $\mathrm{X}$ & $\mathrm{X}$ & $\mathrm{X}$ & $\mathrm{X}$ \\
\hline 50 & $X$ & $\mathrm{X}$ & $X$ & $X$ \\
\hline 51 & $\mathrm{X}$ & $\mathrm{X}$ & & $\mathrm{X}$ \\
\hline 53 & & $X$ & $X$ & $X$ \\
\hline 54 & $\mathrm{X}$ & $\mathrm{X}$ & $\mathrm{X}$ & $X$ \\
\hline 55 & $\mathrm{X}$ & $\mathrm{X}$ & $X$ & $X$ \\
\hline 72 & & $\mathrm{X}$ & $X$ & $\mathrm{X}$ \\
\hline 73 & & $\mathrm{X}$ & $\mathrm{X}$ & $\mathrm{X}$ \\
\hline 75 & & $X$ & $X$ & $\mathrm{X}$ \\
\hline 76 & $\mathrm{X}$ & $\mathrm{X}$ & $\mathrm{X}$ & $X$ \\
\hline 77 & & $\mathrm{X}$ & $X$ & $\mathrm{X}$ \\
\hline 79 & $\mathrm{X}$ & $X$ & & $X$ \\
\hline
\end{tabular}

Variedades utilizadas previamente por los campesinos no se conoce su procedencia y origen.

\begin{tabular}{lcccc}
\hline Negra CPA & $\mathrm{X} 1$ & $*$ & & $\mathrm{X} 2$ \\
Roja CPA & & & $\mathrm{X}$ & $*$ \\
Blanca CPA & & & $\mathrm{X}$ & $*$ \\
TOTAL & 14 & 20 & 17 & 21 \\
\hline
\end{tabular}

* Por su bajo rendimiento los campesinos no la incluyeron en el análisis.

$\mathrm{X}$ variedad sembrada en la prueba evaluativa en la cooperativa

se concluyó la cosecha de las variedades más tardías. En la CPA J. Dimitrov, en siembras tempranas, se sembraron 17 variedades (15 variedades de la feria y dos variedades utilizadas por la cooperativa de grano rojo y grano de color blanco), cada una en dos surcos de diferentes dimensiones según la cantidad de semilla entregada. La distancia entre surco fue de $60 \mathrm{~cm}$. El 27 de diciembre se terminó de cosechar las variedades más tardías. 
El 8 de enero del 2002 se efectuó un taller en el cual se discutieron los resultados de las siembras tempranas en las CPAs Gilberto León y Jorge Dimitrov, y se intercambiaron algunos materiales entre las cooperativas.

Segunda etapa selectiva de los frijoles en las CPAs: En la CPA G. León se sembraron para su evaluación en siembras tardías 20 variedades (13 de las siembras tempranas de la CPA y siete del intercambio con la otra cooperativa). Cada tres surcos de frijol se intercaló un surco de maíz, la distancia entre surco fue de $80 \mathrm{~cm}$. En la CPA J. Dimitrov se sembraron 23 variedades (17 de las siembras tempranas de la CPA, cinco del intercambio con la otra cooperativa para evaluar en siembras tardías y la variedad negra de la CPA).

Etapas selectivas ejecutadas por los campesinos de la Palma: Las variedades fueron sembradas en sus fincas en dos épocas de siembra (Cuadro 2), temprana (agosto-octubre) y tardía (diciembre-febrero). Las siembras se efectuaron según la forma tradicional de siembra por surcos y fueron incluidas las varieda-

Cuadro 2. Campesinos experimentadores de La Palma, fechas de siembra de los estudios evaluativos y los genotipos evaluados. El Tejar La Jocuma, Cuba, 2001 y 2002

\begin{tabular}{|c|c|c|c|c|c|c|c|c|c|c|c|c|c|c|}
\hline \multirow[t]{2}{*}{ No } & \multirow[t]{2}{*}{ Campesino } & \multirow{2}{*}{$\begin{array}{c}\text { Fecha } \\
\text { siembra } \\
\text { Variedades }\end{array}$} & \multicolumn{9}{|c|}{$\begin{array}{c}\text { Número que identifica las variedades } \\
\text { seleccionadas en la Feria }\end{array}$} & \multicolumn{3}{|c|}{$\begin{array}{l}\text { Variedades utilizadas previamente } \\
\text { por los campesinos }\end{array}$} \\
\hline & & & 41 & 48 & 50 & 54 & 55 & 56 & 60 & 63 & & T-N & T-R & T-B \\
\hline \multirow[t]{3}{*}{1} & Sergio y Vestina & $21 / 10 / 01$ & $\mathrm{X}$ & $\mathrm{X}$ & $\mathrm{X}$ & $\mathrm{X}$ & $\mathrm{X}$ & $\mathrm{X}$ & $\mathrm{X}$ & $\mathrm{X}$ & & $\mathrm{X}$ & - & $X$ \\
\hline & & $15 / 1 / 02$ & $\mathrm{X}$ & $\mathrm{X}$ & $\mathrm{X}$ & $\mathrm{X}$ & $\mathrm{X}$ & $\mathrm{X}$ & $\mathrm{X}$ & $\mathrm{X}$ & & $\mathrm{X}$ & - & $\mathrm{X}$ \\
\hline & & Variedades & 9 & 11 & 13 & 15 & 25 & 43 & 50 & 72 & 77 & $\mathrm{~T}-\mathrm{N}$ & $\mathrm{T}-\mathrm{R}$ & $\mathrm{T}-\mathrm{B}$ \\
\hline \multirow[t]{3}{*}{2} & Silvia Hernández & $28 / 8 / 01$ & $\mathrm{X}$ & $\mathrm{X}$ & $X$ & $\mathrm{X}$ & $\mathrm{X}$ & $\mathrm{X}$ & $\mathrm{X}$ & - & - & $\mathrm{X}$ & $\mathrm{X}$ & - \\
\hline & & $29 / 12 / 01$ & $\mathrm{X}$ & $\mathrm{X}$ & $\mathrm{X}$ & $\mathrm{X}$ & $X$ & - & $\mathrm{X}$ & $\mathrm{X}$ & $X$ & - & - & - \\
\hline & & Variedades & 6 & 8 & 45 & 55 & 74 & & & & & $\mathrm{~T}-\mathrm{N}$ & $\mathrm{T}-\mathrm{R}$ & T-B \\
\hline \multirow[t]{3}{*}{3} & Berto Ireno & $18 / 10 / 01$ & $\mathrm{X}$ & $\mathrm{X}$ & $\mathrm{X}$ & $\mathrm{X}$ & $\mathrm{X}$ & & & & & $\mathrm{X}$ & $\mathrm{X}$ & - \\
\hline & & $2 / 2 / 02$ & $\mathrm{X}$ & $\mathrm{X}$ & - & - & - & & & & & - & - & - \\
\hline & & Variedades & 44 & 50 & 58 & 62 & & & & & & $\mathrm{~T}-\mathrm{N}$ & $\mathrm{T}-\mathrm{R}$ & T-B \\
\hline \multirow[t]{2}{*}{4} & Pucho Prieto & $21 / 9 / 01$ & $\mathrm{X}$ & $\mathrm{X}$ & $X$ & $\mathrm{X}$ & & & & & & $\mathrm{X}$ & $\mathrm{X}$ & - \\
\hline & & Variedades & 11 & 18 & 44 & 50 & 54 & 73 & & & & $\mathrm{~T}-\mathrm{N}$ & $\mathrm{T}-\mathrm{R}$ & $\mathrm{T}-\mathrm{B}$ \\
\hline \multirow[t]{3}{*}{5} & Reino Medero & $21 / 10 / 01$ & $\mathrm{X}$ & $\mathrm{X}$ & $\mathrm{X}$ & $\mathrm{X}$ & $\mathrm{X}$ & $\mathrm{X}$ & & & & $\mathrm{X}$ & $\mathrm{X}$ & - \\
\hline & & $17 / 1 / 02$ & $\mathrm{X}$ & - & - & $\mathrm{X}$ & - & $\mathrm{X}$ & & & & - & - & - \\
\hline & & Variedades & 1 & 17 & 21 & 41 & 44 & 77 & 79 & & & $\mathrm{~T}-\mathrm{N}$ & $\mathrm{T}-\mathrm{R}$ & T-B \\
\hline \multirow[t]{3}{*}{6} & Andrés Aldaz & $1 / 9 / 01$ & $\mathrm{X}$ & $\mathrm{X}$ & $\mathrm{X}$ & $\mathrm{X}$ & $\mathrm{X}$ & $\mathrm{X}$ & $\mathrm{X}$ & & & $\mathrm{X}$ & - & - \\
\hline & & $2 / 1 / 02$ & - & $\mathrm{X}$ & $\mathrm{X}$ & $\mathrm{X}$ & $\mathrm{X}$ & $\mathrm{X}$ & $\mathrm{X}$ & & & - & - & - \\
\hline & & Variedades & 5 & 9 & 42 & 47 & 72 & & & & & $\mathrm{~T}-\mathrm{N}$ & $\mathrm{T}-\mathrm{R}$ & $\mathrm{T}-\mathrm{B}$ \\
\hline \multirow[t]{3}{*}{7} & Gervacio Pérez & $13 / 10 / 01$ & $\mathrm{X}$ & $\mathrm{X}$ & $X$ & $\mathrm{X}$ & $\mathrm{X}$ & & & & & $\mathrm{X}$ & $\mathrm{X}$ & - \\
\hline & & $10 / 1 / 02$ & $X$ & $\mathrm{X}$ & $X$ & $\mathrm{X}$ & $\mathrm{X}$ & & & & & - & - & - \\
\hline & & Variedades & 1 & 2 & 3 & 12 & 21 & 41 & 44 & 77 & 79 & T-L & $\mathrm{T}-\mathrm{R}$ & T-B \\
\hline \multirow[t]{2}{*}{8} & Pedro F. (Coco) & $26 / 8 / 01$ & $\mathrm{X}$ & $\mathrm{X}$ & $\mathrm{X}$ & $\mathrm{X}$ & $\mathrm{X}$ & $\mathrm{X}$ & $\mathrm{X}$ & $\mathrm{X}$ & $X$ & $\mathrm{X}$ & - & - \\
\hline & & $14 / 1 / 02$ & $\mathrm{X}$ & $\mathrm{X}$ & $\mathrm{X}$ & $\mathrm{X}$ & $\mathrm{X}$ & $\mathrm{X}$ & $X$ & $X$ & $\mathrm{X}$ & $\mathrm{X}$ & - & - \\
\hline
\end{tabular}

T-L frijol mezclado denominado por el agricultor como ligatrucho.

T-N frijol negro, T-R frijol rojo, T-B frijol blanco utilizados tradicionalmente por los agricultores.

$\mathrm{X}$ variedad incluida en la prueba evaluativa en las fincas de los campesinos. 
des tradicionales de frijol que utilizaban los campesinos. En el 2004 se visitaron las fincas y las CPAs y se evaluó la situación actual existente para conocer la efectividad del proceso de FP.

\section{RESULTADOS Y DISCUSIÓN}

Como resultado de la fase de diagnóstico se encontró en las áreas de intervención del proyecto que más del $85 \%$ de los agricultores no utilizaban la semilla del sistema formal y por tanto las variedades obtenidas por los diversos programas de mejora no llegaban a la mayoría de los agricultores.

Esta aseveración se ha seguido confirmando en otras localidades y sistemas productivos en los resultados de los diagnósticos de otros proyectos (Moreno et al. 2003; Suárez et al. 2005; Hernández et al. 2005 y Ponce et al. 2004).

Según datos estadísticos nacionales (Anuario Estadístico de Cuba 2004), relacionados con el sector agropecuario, más del $80 \%$ de la producción del frijol provenía del sector no estatal (UBPC, CPA, CCS, campesinos dispersos y otros privados). Estas producciones en general tienen bajo potencial de rendimiento, debido entre otros factores a la baja calidad de la semilla, baja diversidad varietal y la poca disponibilidad. Por tanto si se cumplen porcentajes semejantes a los encontrados en el diagnóstico de este trabajo, se puede estimar que mucho más de la mitad de esta producción obtenida por cientos de miles de agricultores en todo el país, en más de 3.000 estructuras organizativas de producción, no están cubiertos por el sistema formal de semilla. Estos agricultores representan la mayoría, por lo tanto, el buscar un mecanismo que resuelva en parte este déficit de semilla, el aumento de la calidad de la semilla en ese sistema y el aumento de la diversidad, es de vital importancia para Cuba. En tal sentido, se presenta un posible mecanismo alternativo para resolver el déficit de semilla existente en Cuba.

A partir de las colectas en las localidades donde se ha intervenido en el proceso, se ha aumentado la disponibilidad de mayor diversidad, a través de las ferias, siendo su efectividad muy parecida a las variedades comerciales y pre-comerciales; por tal motivo, existe un impacto positivo de las colectas en su magnitud y diversidad.

En el Cuadro 3 se presenta el total de las colectas logradas en este periodo de investigación: en frijol se han colectado 136 accesiones de los campesinos y recibido de donaciones 162 accesiones procedentes de diversas colecciones "ex situ" de centros de investigaciones, esto da un total de 298 accesiones posibles a ofertar en las ferias de diversidad. Además se han colectado y se han utilizado de las colecciones "ex situ" más de 400 accesiones de otros cultivos (maíz, arroz, caupí, trigo, triticale, soya, sorgo, habichuela, yuca y papa). Como se observa, se ha logrado aumentar la disponibilidad de la diversidad genética en esta especie. Estas colectas se seleccionaron negativamente, con base en susceptibilidad a las plagas y enfermedades, las restantes son multiplicadas e ingresadas a las colecciones de trabajo, y son difundidas en las ferias de diversidad. Se logró un impacto sustancial en el aumento de la diversidad genética por este procedimiento.

Viñals (2001) y Viñals et al. (2002) observaron que los campesinos pueden jugar un papel importante en el mejoramiento de las plantas, ya que las variedades que ellos han seleccionado y utilizan pueden superar a las variedades comerciales y precomerciales en algunos componentes del rendimiento.

Cuadro 3. Magnitud de las colectas criollas y acriolladas colectadas de frijol en diversas localidades del país o donadas por otros centros de investigaciones, Cuba, 1999 al 2004.

\begin{tabular}{cccc}
\hline Año & $\begin{array}{c}\text { Variedades } \\
\text { colectadas }\end{array}$ & $\begin{array}{c}\text { Germoplasma } \\
\text { de otros centros }\end{array}$ & $\begin{array}{c}\text { Total } \\
\text { general }\end{array}$ \\
\hline 1999 & 50 & $17^{*}$ & 67 \\
2000 & 6 & $60^{*}$ & 66 \\
2001 & 5 & $50^{* *}$ & 55 \\
2002 & 3 & - & 3 \\
2003 & 45 & $35^{* * *}$ & 80 \\
2004 & 27 & - & 27 \\
Total & 136 & 162 & 298 \\
\hline
\end{tabular}

*Instituto de Investigaciones Fundamentales de Agricultura Tropical.

**Instituto de Investigaciones de Suelos.

****Estación Territorial de Investigaciones Agropecuarias de Holguín. Las variedades comerciales fueron donadas por la Empresa de Semilla y el Instituto Hortícola Liliana Dimitrova 
En el marco del proyecto de FP ha existido un movimiento organizado y sostenido de Ferias de Agrobiodiversidad en las dos provincias más occidentales del país, en el cultivo del frijol. Estas se han ido además ejecutando en diversas provincias, además han sido adoptadas por diversos centros de investigación cubanos. Hasta cierre del 2004 en todo el país se habían ejecutado en el cultivo del frijol 18 ferias de diversidad (Cuadro 4); se han ejecutado 67 ferias de otros cultivos que no se incluyen en este cuadro, lo que da un total general de 85 ferias ejecutadas en Cuba.

$\mathrm{Al}$ inicio como se ve, se ejecutaron las ferias dentro de los centros de investigación con un total de cuatro ferias, dos de ellas en el INCA entre el 1999 y 2002, y dos en la ETIAH, en el 2003. Posteriormente se planificaron 14 ferias en localidades campesinas; dos de ellas ya han sido ejecutadas y organizadas por las comunidades campesinas que demuestra la sostenibilidad de su ejecución por las autoridades municipales.

Como un resultado de su aplicabilidad en el contesto cubano de las Ferias de Diversidad, es el reconocimiento por el "Sistema nacional de producción popular del arroz" en el 2004, las ferias como mecanismo para la difusión de las variedades. Se plantarán ferias de arroz en cada municipio del país para tratar de abarcar a los casi 200.000 productores del grano en todo el país (Alemán 2005).

Al evaluar el impacto de las ferias en cuanto a la difusión de materiales y a número de participantes, se observa en el Cuadro 5 que se han puesto a disposición de los campesinos en cada feria más de 100 materiales, en promedio, siendo seleccionadas 38 de ellas por
Cuadro 4. Evidencias cuantitativas de la magnitud de las ferias de diversidad ejecutadas en diversas localidades de Cuba 2001 al 2004.

\begin{tabular}{|c|c|c|}
\hline \multicolumn{2}{|c|}{ Ubicación de la Feria } & \multirow{2}{*}{ \# de ferias } \\
\hline Centros de investigación & $\begin{array}{l}\text { INCA (1999, } \\
2001 \text { y } 2002)\end{array}$ & \\
\hline & ETIAH (2003) & 2 \\
\hline \multicolumn{2}{|l|}{ Subtotal } & 4 \\
\hline \multirow{3}{*}{$\begin{array}{l}\text { Áreas campesinas organizadas } \\
\text { y financiadas por los centros de } \\
\text { investigación }\end{array}$} & INCA & 3 \\
\hline & CIAP & 5 \\
\hline & ETIAH & 4 \\
\hline \multicolumn{2}{|l|}{ Subtotal } & 12 \\
\hline \multirow{3}{*}{$\begin{array}{l}\text { Áreas campesinas organizadas } \\
\text { y financiadas por las localidades }\end{array}$} & INCA & 2 \\
\hline & CIAP & - \\
\hline & ETIAH & - \\
\hline \multicolumn{2}{|l|}{ Subtotal } & 2 \\
\hline \multicolumn{2}{|l|}{ Total INCA } & 7 \\
\hline \multicolumn{2}{|l|}{ Total CIAP } & 5 \\
\hline \multicolumn{2}{|l|}{ Total ETIAH } & 6 \\
\hline \multicolumn{2}{|c|}{ TOTAL GENERAL } & 18 \\
\hline
\end{tabular}

lo menos una vez; es decir por cada feria ejecutada se entregaron en cada localidad 38 variedades que ampliaron en gran medida la diversidad genética de estas especies en la localidad, porque en todos los casos se a triplicado o cuadriplicado la cantidad de variedades de esa especie que existían en las localidades, previo al desarrollo de las ferias de diversidad, siendo esa nueva diversidad basada en los tres colores del grano (negro, rojo y blanco). Dichas ferias se han ejecutado

Cuadro 5. Impacto de las ferias por difusión de materiales y cantidad de variedades seleccionadas por los campesinos en diversas localidades de Cuba 2001 al 2004.

\begin{tabular}{lccccc}
\hline $\begin{array}{c}\text { Especie } \\
\text { Variedades } \\
\text { y localidades }\end{array}$ & $\begin{array}{c}\text { Promedio } \\
\text { variedades } \\
\text { ofertadas }\end{array}$ & $\begin{array}{c}\text { Promedio } \\
\text { variedades } \\
\text { seleccionadas }\end{array}$ & Localidades & $\begin{array}{c}\text { Total } \\
\text { participantes }\end{array}$ & $\begin{array}{c}\text { Total } \\
\text { seleccionadores }\end{array}$ \\
\hline & & & 4 & 512 & 222 \\
INCA & 93 & 52 & 4 & 408 & 119 \\
UCLV & 118 & 37 & 5 & 546 & 273 \\
ETIAH & 105 & 24 & 13 & 1.466 & 694 \\
\hline TOTAL & 105 & 38 & & & \\
\hline
\end{tabular}


en 13 localidades de varias provincias del país. A estas ferias han asistido más de 1.466 pobladores de las comunidades campesinas, siendo seleccionadores en las ferias más de 690 campesinos, lo que demuestra la amplitud de la red experimental ya que ellos evalúan en sus fincas los materiales seleccionados en las ferias. En el Cuadro 6 se presenta el número promedio de variedades criollas o acriolladas ofertadas y seleccionadas en las 18 ferias ejecutadas de frijol; 132 materiales procedentes de colectas se han utilizado en las ferias ejecutadas y de ellas se han seleccionado por los agricultores 53 para un $40 \%$ de efectividad, valor muy parecido al promedio general logrado, lo que demuestra el valor potencial de estos materiales. Según datos del Cuadro 2, la suma de las colectas llegaron a 136, y de estas se han utilizado 132 para un $97 \%$ de utilización del material prospectado hasta el presente.

Cuadro 6. Promedio de variedades criollas o acriolladas seleccionadas, efectividad de las mismas en las ferias en diversas localidades de Cuba 2001 al 2004.

\begin{tabular}{|c|c|c|c|c|c|}
\hline \multirow{3}{*}{$\begin{array}{c}\text { Especies/ } \\
\text { Origen }\end{array}$} & \multirow{3}{*}{$\begin{array}{c}\text { Cant. de } \\
\text { Ferias }\end{array}$} & \multicolumn{4}{|c|}{ Frijol } \\
\hline & & \multicolumn{2}{|c|}{ Criollas } & \multirow{2}{*}{$\begin{array}{c}\text { Otras } \\
\text { ofertadas }\end{array}$} & \multirow{2}{*}{$\begin{array}{l}\text { Otras } \\
\text { selecc. }\end{array}$} \\
\hline & & Ofertadas & Selecc & & \\
\hline INCA & 6 & 63 & 37 & 20 & 15 \\
\hline UCLV & 6 & 38 & 13 & 71 & 26 \\
\hline ETIAH & 6 & 31 & 3 & 64 & 21 \\
\hline TOTAL & 18 & 132 & 53 & 155 & 62 \\
\hline
\end{tabular}

Las ferias son la interfase necesaria para la extensión y generalización de las variedades de los diversos programas de mejoramiento. El solo hecho de ejecutar la feria posibilita la introducción a la comunidad de una amplia diversidad. Los datos de los resultados de más de 85 ferias ejecutadas en diversos cultivos, indican que como promedio más del $35 \%$ de la diversidad expuesta se logra dejar en la comunidad.

También se ha encontrado que más del $85 \%$ de los participantes logran con las variedades seleccionadas por ellos, un diferencial de selección positivo con respecto a la media del rendimiento de cada Feria (Ponce et al. 2003); además se ha demostrado, que es una herramienta eficiente, para de forma rápida, posibilitar que las variedades del sistema formal sean introducidas en las comunidades.

Ningún otro programa de mejoramiento puede llegar con tanta amplitud a la finca de cada agricultor; por otro lado, las limitantes en insumos actuales acentúan que cada finca se convierte a un ecosistema diferente. En el Cuadro 5 se observa que 694 campesinos han elegido grupos de variedades y las han llevado a sus fincas para llevar a cabo la selección definitiva de los materiales que utilizarán para la producción. Este método de FP ayuda a la selección de las variedades con respuesta específica que favorece la interacción del genotipo - ambiente que es un grave problema en los programas centralizados cuando se logra seleccionar materiales con amplia respuesta bajo paquetes tecnológicos precisos con todos los insumos garantizados, lo cual es más la excepción que la regla en las condiciones cubanas (Ortiz et al. 2003 a; Ortiz et al. 2003 b).

Un ejemplo de la importancia de la respuesta específica, son los resultados encontrados en CPAs de San Antonio de los Baños. Estas cooperativas colindan y los campos donde se evaluaron las variedades, no estaban a mas de $600 \mathrm{~m}$, en el mismo tipo de suelo y fueron sembrados con seis días de diferencia en la siembra temprana del 2001 (Cuadro 1), la CPA Gilberto León sembró el frijol intercalado en una plantación nueva de plátano burro y la distancia de los surcos fue de $80 \mathrm{~cm}$; y la CPA Jorge Dimitrov sembró en monocultivo a una distancia entre surcos de $60 \mathrm{~cm}$. Esto determinó una respuesta diferenciada en un grupo importante de variedades.

La comparación de los resultados en el 2002, en siembras tardías de las nueve variedades que se intercambiaron entre las cooperativas evidencia también una alta influencia de la interacción de las tecnologías con las variedades. En la siembras tardías, los campos no estaban a más de $400 \mathrm{~m}$, eran del mismo tipo de suelo y fueron sembrados solamente con cuatro días de diferencia, pero en la CPA Gilberto León se sembró el frijol intercalado con maíz y la distancia de los surcos fue de $80 \mathrm{~cm}$ y en la CPA Jorge Dimitrov se sembró en monocultivo y los surcos a $60 \mathrm{~cm}$. Esto determinó igualmente una respuesta diferenciada en un grupo importante de variedades. 
El análisis del modelo AMMI efectuado con los datos de rendimiento de las seis variedades que se repitieron en las dos cooperativas en siembras tempranas y tardías $(42,44,48,50,54$, y 55), presentaron un alto efecto de interacción variedad x (época - CPA) (Figura 1). En este caso el primer eje presentó el $58,8 \%$ y el segundo eje el $31,2 \%$ de la variación. Se aprecia que las variedades 44, 54 y 55 interactúan positivamente en $\mathrm{E}_{1} \mathrm{~F}_{1}$ y negativamente en $\mathrm{E}_{1} \mathrm{~F}_{2}$. La variedad 50 interactúa positivamente en $\mathrm{E}_{2} \mathrm{~F}_{2}$ y negativamente en $\mathrm{E} 2 \mathrm{~F} 1$, mientras las variedades 42 y 48 interactúan positivamente en $\mathrm{E}_{1} \mathrm{~F}_{2}$ y negativamente en $\mathrm{E}_{1} \mathrm{~F}_{1}$ y $\mathrm{E}_{2} \mathrm{~F}_{2}$. De manera general se puede apreciar un gran efecto de la interacción variedad x (época - CPA).

Los resultados anteriores reafirman, la importancia de la selección de las variedades bajo las condiciones específicas de las fincas y sus tecnologías, hecho que sólo se logra a través de la experimentación campesina en la finca, en correspondencia con lo señalado en la literatura en cuanto a la importancia de seleccionar las variedades en el mismo ecosistema donde se explotarán.

A continuación se presentan resultados actualizados del efecto de las ferias y la experimentación campesina sobre la producción del frijol en las fincas y cooperativas: bajo seguimiento de los datos de las primeras ferias con campesinos de La Palma, Pinar del Río y de las cooperativas de la Habana.
Como se observa en la Figura 2 existió un efecto importante después de cuatro años de la acción del FP, en cuanto a la proporción de las variedades preseleccionadas en las ferias y seleccionadas definitivamente en la finca o cooperativa. Las variedades del FP han sido estables, ya que después de cuatro años los agricultores las siembran en alta proporción en las fincas o cooperativas. El grano de color negro abarca entre el 60 y el $80 \%$ en La Palma y San Antonio, respectivamente, y en los rojos y blancos las siembras abarcan entre el 84 y $100 \%$.

En la Figura 3 se observa un efecto sostenido y muy importante después de cuatro años de la aplicación del FP en cuanto al aumento de la diversidad, que pasó en el caso de las fincas individuales de 2,29

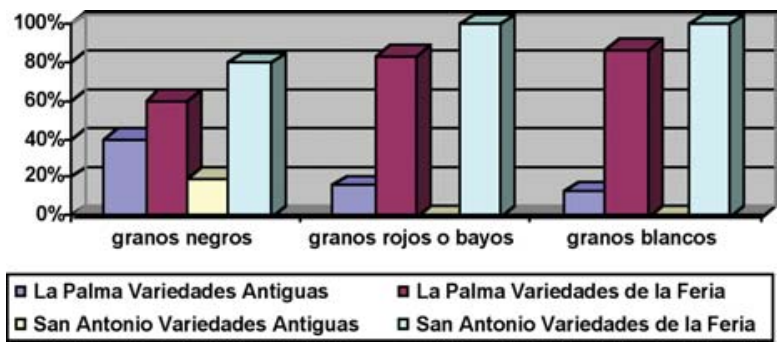

Figura 2. Origen de las variedades utilizadas después de cinco años de intervención del proyecto en La Palma y San Antonio de los Baños, Cuba. 2000 y 2004.

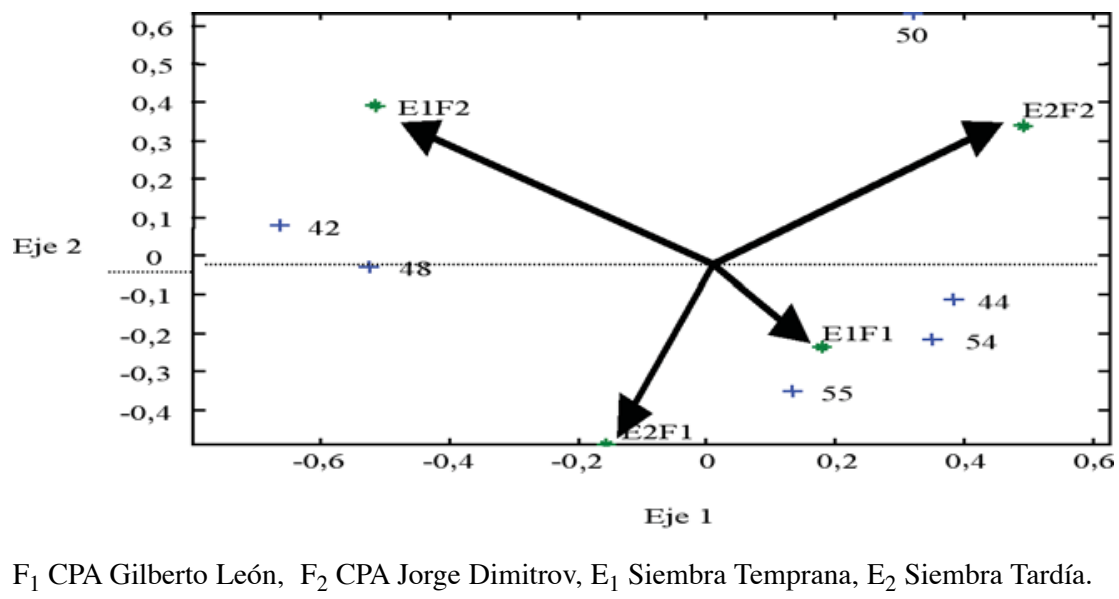

Figura 1. Puntuaciones de los ejes 1 y 2 del Biplot en las variedades y combinaciones de época x CPA), San Antonio de los Baños, Cuba, 2001 y 2002. 


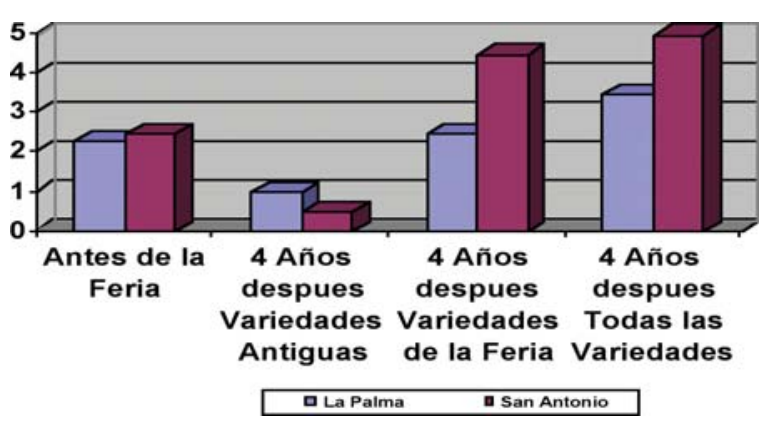

Figura 3. Variedades por fincas y cooperativas antes y después de las Ferias de diversidad y la experimentación campesina en La Palma y San Antonio de los Baños, Cuba. 2000 y 2004.

variedades por finca a 3,43 donde las variedades seleccionadas por los campesinos mantienen una gran proporción (70,8\%); además las variedades antiguas utilizadas por los campesinos, aunque han disminuido su proporción se mantienen en una aceptable proporción $(29,2 \%)$.

En las CPAs de San Antonio de los Baños se pasó de 2,5 variedades por cooperativa a 5,0, y las variedades seleccionadas por los campesinos se mantuvo una gran proporción (90,0\%); además, las variedades antiguas utilizadas por las cooperativas, han disminuido su proporción a sólo un $10 \%$. La ampliación de la diversidad es superior en las CPAs que las que se presentan en las fincas de los campesinos individuales de las CCS de La Palma.

Como se observa (Cuadro 7) hubo un aumento sustancial del rendimiento en todas las áreas con la aplicación del FP, por tanto se puede afirmar que con

Cuadro 7. Comportamiento del rendimiento del cultivo de frijol ( $t / h a)$ en fincas y cooperativas en La Palma y San Antonio de los Baños, Cuba, 2000 al 2004.

\begin{tabular}{lcccc}
\hline & $\mathbf{2 0 0 0 / 0 1}$ & $\mathbf{0 1} / \mathbf{0 2}$ & $\mathbf{0 2} / \mathbf{0 3}$ & $\mathbf{0 3 / 0 4}$ \\
\hline $\begin{array}{l}\text { San Antonio de los Baños } \\
\quad\end{array}$ & 0,37 & 0,55 & 0,46 & 0,80 \\
$\quad$ CPA G. León & 0,43 & 0,49 & 0,69 & 1,21 \\
$\quad$ CPA J. Dimitrov & & & & \\
\hline $\begin{array}{l}\text { La Palma (promedio de las } \\
\text { ocho fincas) }\end{array}$ & 0,59 & 0,63 & 0,74 & 0,93 \\
\hline
\end{tabular}

ISSN: $1021-7444$ el aumento de la diversidad, aumentó el rendimiento y además aumentó el área dedicada a este cultivo.

\section{AGRADECIMIENTOS}

El apoyo para la conducción de las actividades de fitomejoramiento participativo en frijol en Cuba, durante el periodo de 2001 al 2004, que fue proporcionado por el IDRC de Canadá y el Ministerio de Educación Superior de Cuba y a partir del 2003 al 2004 se le sumaron los fondos de COSUDE de Suiza.

Agradecemos además, a todos los campesinos de las CPA Gilberto León y Jorge Dimitrov de San Antonio de los Baños en La Habana y los campesinos del El Tejar La Jocuma - en La Palma, Pinar del Río todo su trabajo experimental ejecutado y su alto desprendimiento.

\section{LITERATURA CITADA}

ALEMÁN, M. 2005. Documental sobre proceso de fitomejoramiento participativo en arroz en Cuba "ARROZ CON AMOR SE PAGA”, película en una video cinta y soporte electrónico, La Palma producciones, La Habana, Cuba.

ANUARIO ESTADÍSTICO DE CUBA. 2004. Organización Nacional de Estadística (ONE), edición 2005. p. 189218.

DE LA FE, C.; RIOS, H.; ORTIZ, R.; MARTINEZ, M.; ACOSTA, R.; PONCE M.; MIRANDA, S.; MORENO, I.; MARTIN, L. 2003. Las ferias de agrodiversidad. Guía metodológica para la organización y desarrollo en Cuba. Cultivos Tropicales 24(4): 95-106.

HERNÁNDEZ, L; PINO, M. A.; CALVES, E.; DOMINI, M. E.; RAMÍREZ, A.; TERÁN, Z. 2005. Caracterización de los agricultores, biodiversidad y tecnologías de cultivos en el consejo popular, norte y sur del municipio de San José de las Lajas, provincia de La Habana. Cultivos Tropicales 26(3): 11-16.

MORENO, I.; RÍOS, H.; ALMEKINDERS, C. 2003. Caracterización de los sistemas locales de arroz de la Palma, Pinar del Río. Cultivos Tropicales 24(4): 49-54. 
ORTIZ, R.; PONCE, M.; RÍOS, H.; VERDE, G.; ACOSTA, R.; MIRANDA, S.; MARTIN, L.; MORENO, I.; MARTÍNEZ, M.; DE LA FE, C.; VARELA, M. 2003a. Efectividad de la experimentación campesina en la microlocalización de variedades de frijol y la evaluación genotipo-ambiente. Cultivos Tropicales 24:107-114.

ORTIZ, R.; PONCE, M.; RÍOS, H.; VERDE, G.; ACOSTA, R.; MIRANDA, S.; MARTIN, L.; MORENO, I.; MARTÍNEZ, M.; DE LA FE, C.; VARELA, M. 2003b. Impactos de la experimentación campesina en cooperativas de producción de la Habana. Cultivos Tropicales 24:115-122.

PONCE, M.; ORTIZ, R.; RÍOS, H.; DE LA FE, C.; VERDE G.; MARTÍNEZ, M.; CARBONELL, A.; MARTÍN, A.; ACOSTA, R.; MIRANDA S. 2003 Caracterización de una amplia colección de frijoles y resultados de la selección campesina. Cultivos Tropicales 24(4): 85-88.
PONCE, M.; ORTIZ, R.; RÍOS, H.; DE LA FE, C.; VALDEZ, R.; HERNÁNDEZ, R. 2004. Elaboración de piensos locales por métodos participativos. In: MEMORIAS XIII CONGRESO DEL INCA. San José de las Lajas, Cuba. 178 p.

SUAREZ, L.; HERNÁNDEZ, M. M.; RIOS, H. 2005 Caracterización de los sistemas locales de manejo de la semilla de yuca (Manihot esculenta Crantz) en dos localidades del municipio La Palma, Pinar del Río. Cultivos Tropicales 26 (2): 59-63.

VIÑALS, M. E. 2001. Análisis de la diversidad fenotípica de variedades de frijol (Phaseolus vulgaris L.) manejada por los agricultores. Tesis de Maestría, San José de las Lajas, La Habana, Cuba, UNAH. 79 p.

VIÑALS, M. E.; ORTÍZ, R.; PONCE, M.; RÍOS, H. 2002. Análisis de la diversidad fenotípica de variedades de frijol (Phaseolus vulgaris L.) utilizadas por los campesinos en la comunidad La Palma en Pinar del Río. Cultivos Tropicales 23(1): 34-40. 